\title{
Time Domain Linear Sampling Method for Inverse Scattering Problems with Cracks
}

\author{
Yang Yue ${ }^{1,2}$, Fuming $\mathrm{Ma}^{1}$ and Bo Chen ${ }^{3, *}$ \\ ${ }^{1}$ Institute of Mathematics, Jilin University, Changchun 130012, P.R. China. \\ ${ }^{2}$ School of Science, Jilin Institute of Chemical Technology, Jilin 132022, P.R. China. \\ ${ }^{3}$ College of Science, Civil Aviation University of China, Tianjin 300300, P.R. China.
}

Received 12 April 2021; Accepted (in revised version) 19 July 2021.

\begin{abstract}
The paper analyses time-dependent scattering and inverse scattering problems with cracks. The well-posedness of the forward scattering problem is proved and a modified retarded potential boundary integral equation method is utilised to solve the forward problem. Besides, the inverse scattering problem of finding the cracks by using measured scattered data is considered. A time domain linear sampling method for inverse problems is developed and the blow-up property is proved. The computation scheme is relatively simple and easy to implement. Numerical examples demonstrate the effectiveness of the methods.
\end{abstract}

AMS subject classifications: 35L20, 65M32

Key words: Time domain, inverse scattering problem, cracks, boundary integral equation, linear sampling method.

\section{Introduction}

Scattering and inverse scattering problems with acoustic waves are important subjects in mathematical physics. Usually, the problems can be split into two groups - viz. frequency domain and time domain problems. The frequency domain problems, dealing mainly with the scattering of time harmonic waves, are extensively studied in the past decades [9-11]. The time domain problems, on the other hand, concerns the scattering of time-dependent waves and the wave equation. They are related to time variables naturally arising in practical problems and attracted a growing attention in recent years. Thus [6] investigates the reconstruction of time-dependent point sources and [19] employs the Schrödinger equation with time-dependent method.

This paper considers the scattering of time-dependent acoustic waves on infinite cylinders with cracks in $\mathbb{R}^{2}$ as cross section. The forward scattering problems can be solved by the retarded potential boundary integral equation (RPBIE) method [2, 14, 17, 24], which is based on the classical boundary integral equations $[18,25,26]$. The inverse problem

*Corresponding author. Email address: charli ecb@163.com (B. Chen) 
consists in finding cracks from measured scattered data. The corresponding frequency domain problems are studied by Newton type iteration methods $[21,22]$ based on the domain derivative $[3,28]$, factorisation method [27] and the linear sampling methods $[4,20]$. However, time domain methods are rarely used in inverse problems. In this paper, a linear sampling method (LSM) is applied to a time domain inverse scattering problem. The LSM is relatively fast since it requires neither the iteration nor the forward solver. Moreover, the LSM is easy to implement since no a priori information about the unknown targets is needed. Therefore, it is widely used in frequency domain problems $[1,4,5]$ and time domain problems $[7,8,15,16]$.

Although time domain LSM has been often used in the reconstruction of bounded scatterers $[8,16]$, the analysis of the inverse scattering problem for cracks is not obvious. The basis of the time domain LSM is the blow-up behavior of the indicator function for points approaching the boundary of the obstacle from the inside. The boundary can be shown by plotting an indicator function over the sampling grid. However, the crack has no concepts of inside and outside. Therefore, in this paper, a new test function is proposed and a new proof of the blow-up property is provided.

The outlines of this paper are as follows. Forward and inverse scattering problems and relevant functional spaces are introduced in Section 2. In Section 3, an RPBIE method and the well-posedness of forward scattering problems are provided. In Section 4, we prove the blow-up property of the time domain LSM for an inverse problem. Section 5 is devoted to the numerical implementation of the RPBIE for the forward problem and the time domain LSM for the inverse problem.

\section{Problem Setting}

Let $\rho:[0,1] \rightarrow \mathbb{R}^{2}$ be an injective and piecewise smooth function such that $\Gamma=\{\rho(s)$ : $s \in[0,1]\}$ is a nonintersecting oriented open arc without cusps in $\mathbb{R}^{2}$, and let $\Gamma_{+}$and $\Gamma_{-}$ denote the right and left sides of $\Gamma$, respectively.

We consider the scattering of time-dependent acoustic waves on $\Gamma$ with Dirichlet boundary conditions. The incident wave is chosen as a cylindrical wave [24] defined by the time convolution

$$
u^{i}(x, t ; y):=G(x, t ; y) * \lambda(t)
$$

where

$$
G(x, t ; y)=\frac{H\left(t-c^{-1}|x-y|\right)}{2 \pi \sqrt{t^{2}-c^{-2}|x-y|^{2}}}
$$

is the Green's function of the d'Alembert operator in $\mathbb{R}^{2}, \lambda(t)$ a signal function, $c$ the sound speed in the homogeneous background medium, and $H$ the Heaviside function. In this work, $\lambda(t)$ is causal - i.e. $\lambda(t)=0$ for all $t<0$.

We split the total wave field $u^{\text {tot }}$ into the incident field $u^{i}$ and the scattered field $u$. Then the scattered field $u$ satisfies the equations

$$
c^{-2} \partial_{t t} u-\Delta u=0 \quad \text { in } \Omega \times \mathbb{R},
$$




$$
\begin{array}{ll}
u^{ \pm}=g & \text { on } \Gamma \times \mathbb{R}, \\
u(\cdot, 0)=\partial_{t} u(\cdot, 0)=0 & \text { in } \Omega,
\end{array}
$$

where $\Omega:=\mathbb{R}^{2} \backslash \Gamma, \Delta$ is the Laplacian in $\mathbb{R}^{2}, \partial_{t}=\partial / \partial t, \partial_{t t}=\partial^{2} / \partial t^{2}, g=-u^{i}$ and $u^{ \pm}(x, t)=\lim _{h \rightarrow 0^{+}} u(x \pm h v, t)$ with the unit normal vector $v$ to the right side of $\Gamma$.

The forward scattering problem [FP] is: Given the incident wave (2.1) and an open arc $\Gamma$, determine the scattered field $u$ from (2.2)-(2.4).

The incident curve $\Gamma_{i}$ and the measurement curve $\Gamma_{m}$ are chosen as closed Lipschitz curves surrounding $\Gamma$. Assume that $\Gamma \cap \Gamma_{i}=\varnothing$ and $\Gamma \cap \Gamma_{m}=\varnothing$. In practice, one usually chooses $\Gamma_{i}=\Gamma_{m}$. The sketch of the problem geometry is presented in Fig. 1.

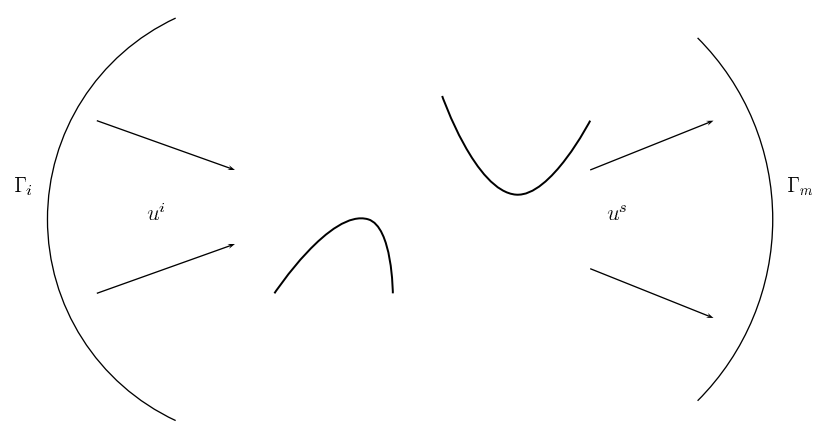

Figure 1: Sketch of the geometry of the problem.

The inverse scattering problem [IP] is: Given the incident wave (2.1) and the measurement data

$$
\left\{u(x, t ; y): x \in \Gamma_{m}, t \in[0, T], y \in \Gamma_{i}\right\},
$$

where $T>0$ is the terminal time, reconstruct the open arc $\Gamma$ from (2.2)-(2.4).

Instead of providing a direct analysis of the open arc, we extend $\Gamma$ to an simply connected, piecewise $C^{1}$ closed curve $\partial D$, which encloses a bounded domain $D \subset \mathbb{R}^{2}$. Assume that the unit outward normal vector $v$ to $\partial D$ coincides with the unit normal vector to the right side of $\Gamma$.

In addition to the Sobolev spaces $L^{2}(\partial D), H^{1 / 2}(\partial D)$ and $H^{-1 / 2}(\partial D)$, more functional spaces on the open $\operatorname{arc} \Gamma \subset \partial D$ are needed - cf. $[4,18,20]$. Define

$$
H^{\frac{1}{2}}(\Gamma):=\left\{\left.\phi\right|_{\Gamma}: \phi \in H^{\frac{1}{2}}(\partial D)\right\}
$$

and denote by $\tilde{H}^{-1 / 2}(\Gamma)$ the dual space of $H^{1 / 2}(\Gamma)$. Moreover, let

$$
\tilde{H}^{\frac{1}{2}}(\Gamma):=\left\{\phi \in H^{\frac{1}{2}}(\Gamma): \operatorname{supp} \phi \subseteq \bar{\Gamma}\right\}
$$

and $H^{-1 / 2}(\Gamma)$ be the dual space of $\tilde{H}^{1 / 2}(\Gamma)$.

Let us recall the definition of the space-time Sobolev spaces $[7,24]$. Let

$$
\begin{aligned}
& \mathbb{C}_{\sigma}:=\{\omega \in \mathbb{C}: \operatorname{Im}(\omega)>\sigma>0\}, \\
& \mathscr{L}^{\prime}(H):=\left\{\phi \in \mathscr{D}^{\prime}(H): \mathrm{e}^{-\sigma t} \phi \in \mathscr{S}^{\prime}(H)\right\},
\end{aligned}
$$


where $\sigma \in \mathbb{R}, H$ is a Hilbert space, $\mathscr{D}^{\prime}(H)$ and $\mathscr{S}^{\prime}(H)$ are the spaces of $H$-valued and tempered distributions on the real line, respectively.

In this work we use the Fourier-Laplace transform

$$
\mathscr{L}[\phi](\omega):=\int_{-\infty}^{\infty} \mathrm{e}^{\mathrm{i} \omega t} \phi(t) \mathrm{d} t, \quad \omega \in \mathbb{C}_{\sigma}
$$

instead of the Fourier transform. Consider the space

$$
H_{\sigma}^{p}(\mathbb{R}, H):=\left\{f \in \mathscr{L}^{\prime}{ }_{\sigma}(H): \int_{-\infty+\mathrm{i} \sigma}^{\infty+\mathrm{i} \sigma}|\omega|^{2 p}\|\mathscr{L}[f](\omega)\|_{H}^{2} \mathrm{~d} \omega<\infty\right\}
$$

equipped with the norm

$$
\|f\|_{H_{\sigma}^{p}(\mathbb{R}, H)}:=\left(\int_{-\infty+\mathrm{i} \sigma}^{\infty+\mathrm{i} \sigma}|\omega|^{2 p}\|\mathscr{L}[f](\omega)\|_{H}^{2} \mathrm{~d} \omega\right)^{\frac{1}{2}},
$$

where $\sigma \in \mathbb{R}, p \in \mathbb{R}$ and define $L_{\sigma}^{2}(\mathbb{R}, H):=H_{\sigma}^{0}(\mathbb{R}, H)$.

\section{Forward Scattering Problem}

According to [24], the time domain analysis depends on the retarded single layer potential

$$
\left(S L_{\partial D} \phi\right)(x, t):=\int_{\mathbb{R}} \int_{\partial D} G(t-\tau, x ; y) \phi(\tau, y) \mathrm{d} s_{y} \mathrm{~d} \tau, \quad x \in \mathbb{R}^{2} \backslash \partial D, \quad t \in \mathbb{R}
$$

and the retarded double layer potential

$$
\left(D L_{\partial D} \phi\right)(x, t):=\int_{\mathbb{R}} \int_{\partial D} \frac{\partial G(t-\tau, x ; y)}{\partial v(y)} \phi(\tau, y) \mathrm{d} s_{y} \mathrm{~d} \tau, \quad x \in \mathbb{R}^{2} \backslash \partial D, \quad t \in \mathbb{R} .
$$

Besides, our analysis also relies on the single layer operator

$$
\left(S_{\partial D} \phi\right)(x, t):=\int_{\mathbb{R}} \int_{\partial D} G(t-\tau, x ; y) \phi(\tau, y) \mathrm{d} s_{y} \mathrm{~d} \tau, \quad x \in \partial D, \quad t \in \mathbb{R} .
$$

Let $[\gamma \gamma u]$ and $\left[\left[\partial_{v} u\right]\right]$ refer to the jumps of $u$ and $\partial u / \partial v$, respectively, defined by

$$
\left[[\gamma u]:=u^{-}-u^{+}, \quad\left[\left[\partial_{v} u\right]:=\frac{\partial u^{-}}{\partial v}-\frac{\partial u^{+}}{\partial v},\right.\right.
$$

where

$$
\frac{\partial u^{ \pm}}{\partial v}=\lim _{h \rightarrow 0^{+}} v \cdot \nabla u(x \pm h v, t), \quad x \in \partial D
$$


Considering an artificial Dirichlet boundary on $\partial D \backslash \Gamma$, the problem [FP] can be reformulated as an equivalent problem [FP2], viz.

$$
\begin{array}{ll}
c^{-2} \partial_{t t} u-\Delta u=0 & \text { in }\left(\mathbb{R}^{2} \backslash \partial D\right) \times \mathbb{R}, \\
u^{ \pm}=g & \text { on } \Gamma \times \mathbb{R}, \\
u^{ \pm}=\gamma u & \text { on }(\partial D \backslash \Gamma) \times \mathbb{R}, \\
u(\cdot, 0)=\partial_{t} u(\cdot, 0)=0 & \text { in } \mathbb{R}^{2} \backslash \partial D,
\end{array}
$$

where $\gamma u$ is the restriction of the real wave field on $(\partial D \backslash \Gamma) \times \mathbb{R}$. Since the crack is located on $\Gamma$, both $u$ and $\partial_{\nu} u$ are continuous across $\partial D \backslash \Gamma$, i.e. $[[\gamma u]]=\left[\left[\partial_{\nu} u\right]\right]=0$ on $\partial D \backslash \Gamma$.

The Kirchhoff's formula - cf. [24],

$$
u=S L_{\partial D}\left[\left[\partial_{\nu} u\right]-D L_{\partial D}[[\gamma u]] \text { in }\left(\mathbb{R}^{2} \backslash \partial D\right) \times \mathbb{R}\right.
$$

and the fact that $[[\gamma u]]=0$ on $\partial D$ yield

$$
u=S L_{\partial D}\left[\left[\partial_{\nu} u\right]\right] \text { in }\left(\mathbb{R}^{2} \backslash \partial D\right) \times \mathbb{R} .
$$

Moreover, since $\left[\left[\partial_{v} u\right]\right]=0$ on $\partial D \backslash \Gamma$, we obtain that

$$
u=S L_{\Gamma}\left[\left[\partial_{\nu} u\right] \quad \text { in }\left(\mathbb{R}^{2} \backslash \Gamma\right) \times \mathbb{R}\right.
$$

is a solution to the problem [FP2]. According to the boundary condition, $\left.\phi=\llbracket\left[\partial_{\nu} u\right]\right]$ satisfies the boundary integral equation

$$
S_{\Gamma} \phi=g \quad \text { on } \Gamma \times \mathbb{R} .
$$

The RPBIE method for the forward scattering problem is: Solve the boundary integral equation (3.2) for $\phi=\left[\left[\partial_{v} u\right]\right.$. Then the scattered field is given by the representation (3.1). sition.

The well-posedness of the forward scattering problem is given by the following propo-

Proposition 3.1. Let $\sigma>0 p \in \mathbb{R}$ and $g \in H_{\sigma}^{p}\left(\mathbb{R}, H^{1 / 2}(\Gamma)\right)$.

(a) The operator $S L_{\Gamma}$ is bounded from $H_{\sigma}^{p}\left(\mathbb{R}, \tilde{H}^{-1 / 2}(\Gamma)\right)$ to $H_{\sigma}^{p-1}\left(\mathbb{R}, H^{1}(\Omega)\right)$ and $S_{\Gamma}$ is bounded from $H_{\sigma}^{p}\left(\mathbb{R}, \tilde{H}^{-1 / 2}(\Gamma)\right)$ to $H_{\sigma}^{p-1}\left(\mathbb{R}, H^{1 / 2}(\Gamma)\right)$.

(b) The operator $S_{\Gamma}$ is invertible. Moreover, the operator $S_{\Gamma}^{-1}$ is bounded from $H_{\sigma}^{p}\left(\mathbb{R}, H^{1 / 2}(\Gamma)\right)$ to $H_{\sigma}^{p-2}\left(\mathbb{R}, \tilde{H}^{-1 / 2}(\Gamma)\right)$.

(c) The forward scattering problem [FP] has a unique solution $u \in H_{\sigma}^{p-3}\left(\mathbb{R}, H^{1}(\Omega)\right)$. Moreover, there exists a constant $C_{\sigma, \Gamma}$ depending only on $\sigma$ and $\Gamma$ such that

$$
\|u\|_{H_{\sigma}^{p-3}\left(\mathbb{R}, H^{1}(\Omega)\right)} \leq C_{\sigma, \Gamma}\|g\|_{H_{\sigma}^{p}\left(\mathbb{R}, H^{1 / 2}(\Gamma)\right)} .
$$

Proof. We consider the claims one by one. 
(a) The proof is similar to the proof of [8, Lemma 6] when $\Gamma$ is the boundary of a bounded domain. A small difference occurs for functional spaces when $\Gamma$ is an open arc but we omit the details.

(b) The analysis similar to [24, Proposition 2.6.1] shows that $S_{\Gamma}$ is invertible and

$$
\left\|\left(\mathscr{L}\left[S_{\Gamma}^{-1}\right] \phi\right)(\omega)\right\|_{\tilde{H}^{-1 / 2}(\Gamma)} \leq C_{\sigma, \Gamma}^{\prime}|\omega|^{2}\|\phi\|_{H^{1 / 2}(\Gamma)},
$$

where $\phi \in H^{1 / 2}(\Gamma), C_{\sigma, \Gamma}^{\prime}$ is a constant depending only on $\sigma$ and $\Gamma$. Fourier transform arguments and [8, Lemma 2] give that $S_{\Gamma}^{-1}$ is bounded from $H_{\sigma}^{p}\left(\mathbb{R}, H^{1 / 2}(\Gamma)\right)$ to $H_{\sigma}^{p-2}\left(\mathbb{R}, \tilde{H}^{-1 / 2}(\Gamma)\right)$. The proof still holds in spite of the difference on the functional spaces $-\mathrm{cf}$. [23].

(c) The uniqueness result of [24, Proposition 2.4.1] still holds for open $\operatorname{arc} \Gamma$, so that $u=S L_{\Gamma} S_{\Gamma}^{-1} g$ is the unique solution to the problem [FP].

\section{The LSM for the Inverse Problem}

The LSM is based on the near field operator and the test function. The near field operator $\mathscr{N}: H_{\sigma}^{p}\left(\mathbb{R}, \tilde{H}^{-1 / 2}\left(\Gamma_{i}\right)\right) \rightarrow H_{\sigma}^{p-4}\left(\mathbb{R}, H^{1 / 2}\left(\Gamma_{m}\right)\right)$ is defined by

$$
\mathscr{N}:=S L_{\Gamma} S_{\Gamma}^{-1} S L_{\Gamma_{i}}^{\lambda}
$$

where $\sigma>0, p \in \mathbb{R}, S L_{\Gamma_{i}}^{\lambda}:=-2 S L_{\Gamma_{i}} * \lambda$ and $\lambda$ is the same signal function as in (2.1) [7]. Note that full definitions of the operators here should include certain trace operators, which are not explicitly given for the sake of simplicity. Formally, the explicit representation of the near field operator is

$$
(\mathscr{N} g)(x, t)=\int_{\mathbb{R}} \int_{\Gamma_{i}} u(x, t-\tau ; y) g(y, \tau) \mathrm{d} s_{y} \mathrm{~d} \tau, \quad x \in \mathbb{R}^{2} \backslash \Gamma, \quad t \in \mathbb{R} .
$$

The test function is defined as

$$
\varphi_{z, \mu}(x, t):=\int_{\mathbb{R}} G(x, t-\mu-\tau ; z) \zeta(\tau) \mathrm{d} \tau, \quad x \in \mathbb{R}^{2} \backslash\{z\}, \quad t \in \mathbb{R},
$$

where $\mu \in \mathbb{R}$ is a time shift, $\zeta(t)$ a continuous function with compact support, and $z \in \mathbb{R}^{2} \backslash \Gamma_{m}$ the sampling point. Then the near field equation is

$$
\mathscr{N} g_{z, \mu}=\varphi_{z, \mu} \quad \text { on } \Gamma_{m} \times \mathbb{R} .
$$

The LSM is to solve the near field equation for $g_{z, \mu}$. The theoretical feasibility of the LSM is based on the blow-up property and auxiliary results are needed for the consequent analysis. 
Lemma 4.1. Let $\sigma>0$ and $p \in \mathbb{R}$. The operators

$$
\begin{aligned}
& S L_{\Gamma}: H_{\sigma}^{p}\left(\mathbb{R}, \tilde{H}^{-1 / 2}(\Gamma)\right) \rightarrow H_{\sigma}^{p-1}\left(\mathbb{R}, H^{1 / 2}\left(\Gamma_{m}\right)\right), \\
& S L_{\Gamma_{i}}^{\lambda}: H_{\sigma}^{p}\left(\mathbb{R}, \tilde{H}^{-1 / 2}\left(\Gamma_{i}\right)\right) \rightarrow H_{\sigma}^{p-1}\left(\mathbb{R}, H^{1 / 2}(\Gamma)\right), \\
& \mathcal{N}: H_{\sigma}^{p}\left(\mathbb{R}, \tilde{H}^{-1 / 2}\left(\Gamma_{i}\right)\right) \rightarrow H_{\sigma}^{p-4}\left(\mathbb{R}, H^{1 / 2}\left(\Gamma_{m}\right)\right)
\end{aligned}
$$

are all bounded, injective and have dense ranges in their image spaces.

Proof. Analysis of the relevant operators for the time domain scattering problem with Dirichlet scatterers is given in $[7,8]$. Similar discussion and Proposition 3.1 leads to the conclusion.

Failing to prove the blow-up property of the near field equation (4.1) directly, we introduce a new test function $\varphi_{L, \mu}$.

Lemma 4.2. Let $\mu \in \mathbb{R}, \sigma>0$ and $\zeta \in H_{\sigma}^{p}\left(\mathbb{R}, \tilde{H}^{-1 / 2}(L)\right)$. Assume that $L$ is a piecewise $C^{1}$, nonintersecting arc without cusps and define

$$
\varphi_{L, \mu}(x, t):=\int_{\mathbb{R}} \int_{L} G(x, t-\mu-\tau ; y) \zeta(y, \tau) \mathrm{d} s_{y} \mathrm{~d} \tau, \quad x \in \mathbb{R}^{2} \backslash \bar{L}, \quad t \in \mathbb{R} .
$$

Then $\varphi_{L, \mu} \in \operatorname{range}\left(S L_{\Gamma}\right)$ if and only if $L \subset \Gamma$.

Proof. According to the definition of the retarded single layer operator, we have

$$
\varphi_{L, \mu}(x, t)=S L_{L} \zeta(x, t-\mu), \quad x \in \mathbb{R}^{2} \backslash \bar{L}, \quad t \in \mathbb{R} .
$$

Assume that $L \subset \Gamma$ and introduce the function

$$
\zeta^{\prime}(x, t):=\left\{\begin{array}{lll}
\zeta(x, t), & x \in L, & t \in \mathbb{R} \\
0, & x \in \Gamma \backslash L, & t \in \mathbb{R} .
\end{array}\right.
$$

Then $\zeta^{\prime} \in H_{\sigma}^{p}\left(\mathbb{R}, \tilde{H}^{-1 / 2}(\Gamma)\right)$ and $\varphi_{L, \mu}(x, t)=S L_{\Gamma} \zeta^{\prime}(x, t-\mu)$, i.e. $\varphi_{L, \mu} \in \operatorname{range}\left(S L_{\Gamma}\right)$.

If $L \not \subset \Gamma$ and $\varphi_{L, \mu} \in \operatorname{range}\left(S L_{\Gamma}\right)$, we have

$$
\varphi_{L, \mu}(x, t)=P(x, t):=\int_{\mathbb{R}} \int_{\Gamma} G(x, t-\tau ; y) \zeta_{2}(y, \tau) \mathrm{d} s_{y} \mathrm{~d} \tau, \quad x \in \Gamma_{m}, \quad t \in \mathbb{R}
$$

for a $\zeta_{2} \in H_{\sigma}^{p}\left(\mathbb{R}, \tilde{H}^{-1 / 2}(\Gamma)\right)$. Then according to $[2,17]$, the Fourier-Laplace transforms

$$
\mathscr{L}\left[\varphi_{L, \mu}\right](x, \omega)=\int_{L} \Phi_{\omega}(x ; y) \hat{\zeta}(y, \omega) \mathrm{d} s_{y}, \quad \mathscr{L}[P](x, \omega)=\int_{\Gamma} \Phi_{\omega}(x ; y) \hat{\zeta}_{2}(y, \omega) \mathrm{d} s_{y}
$$

coincide on $\Gamma_{m}$ for all $\omega \in \mathbb{C}_{\sigma}$, where

$$
\hat{\zeta}=\mathscr{L}[\zeta(x, t-\mu)], \quad \hat{\zeta}_{2}=\mathscr{L}\left[\zeta_{2}(x, t)\right]
$$


and

$$
\Phi_{\omega}(x ; y):=\frac{\mathrm{i}}{4} H_{0}^{(1)}\left(\frac{\omega}{c}|x-y|\right)
$$

is the Green function of the Helmholtz operator $\Delta+(\omega / c)^{2}$ in $\mathbb{R}^{2}$. Since $\Gamma_{m}$ is a closed Lipschitz curve, the unique solvability of the frequency domain problem with the Helmholtz equation and the unique continuation principle implies that $\mathscr{L}\left[\varphi_{L, \mu}\right](\cdot, \omega)$ and $\mathscr{L}[P](\cdot, \omega)$ are equal in $\mathbb{R}^{2} \backslash(\bar{L} \cup \bar{\Gamma})$.

Since $L \not \subset \Gamma$, we conclude that there is a point $x_{0} \in L$ such that $x_{0} \notin \Gamma$ and $\zeta\left(x_{0}, t\right)$ is nontrivial. Let $B_{\varepsilon}\left(x_{0}\right)$ be a small ball centered at $x_{0}$ and such that $B_{\epsilon}\left(x_{0}\right) \cap \Gamma=\varnothing$. Then $\mathscr{L}[P](\omega)$ is analytic in $B_{\epsilon}\left(x_{0}\right)$, while $\mathscr{L}\left[\varphi_{L, \mu}\right](\omega)$ has a singularity at $x_{0}$. This contradicts to $\mathscr{L}\left[\varphi_{L, \mu}\right](\omega)=\mathscr{L}[P](\omega)$. Therefore, $\varphi_{L, \mu} \notin \operatorname{range}\left(S L_{\Gamma}\right)$.

Remark 4.1. In the proof of Lemma 4.2, we noted that there exists a point $x_{0} \in\{x \in L$ : $x \notin \Gamma\}$ such that $\zeta\left(x_{0}, t\right)$ is nontrivial if $L \not \subset \Gamma$. Assume that $\zeta(x, t)=0$ for every point $x \in\{x \in L: x \notin \Gamma\}$ and let $L^{\prime}=L \cap \Gamma$. Then $\zeta(x, t)=0$ for $x \in L \backslash L^{\prime}$ and the test function can be written as

$$
\varphi_{L, \mu}(x, t)=S L_{L^{\prime}} \zeta(x, t-\mu), \quad x \in \mathbb{R}^{2} \backslash\left\{L^{\prime}\right\}, \quad t \in \mathbb{R},
$$

where $L^{\prime} \subset \Gamma$.

The blow-up property of the sampling method with the new test function $\varphi_{L, \mu}$ is based on the following theorem.

Theorem 4.1. Let $\sigma>0, p \in \mathbb{R}$ and $\mu \in \mathbb{R}$. Assume that $\Gamma$ and $L$ are oriented nonintersecting piecewise smooth open arcs without cusps. Then:

(a) For any $\varepsilon>0$, there exists $g_{\mu, \varepsilon}^{L} \in H_{\sigma}^{4}\left(\mathbb{R}, \tilde{H}^{-1 / 2}\left(\Gamma_{i}\right)\right)$ such that

$$
\left\|\mathscr{N} g_{\mu, \varepsilon}^{L}-\varphi_{L, \mu}\right\|_{L_{\sigma}^{2}\left(\mathbb{R}, H^{1 / 2}\left(\Gamma_{m}\right)\right)} \leq \varepsilon .
$$

(b) If $L \not \subset \Gamma$, for $g_{\mu, \varepsilon}^{L} \in H_{\sigma}^{4}\left(\mathbb{R}, \tilde{H}^{-1 / 2}\left(\Gamma_{i}\right)\right)$ such that

$$
\lim _{\varepsilon \rightarrow 0}\left\|\mathscr{N} g_{\mu, \varepsilon}^{L}-\varphi_{L, \mu}\right\|_{L_{\sigma}^{2}\left(\mathbb{R}, H^{1 / 2}\left(\Gamma_{m}\right)\right)}=0
$$

we have

$$
\lim _{\varepsilon \rightarrow 0}\left\|g_{\mu, \varepsilon}^{L}\right\|_{H_{\sigma}^{4}\left(\mathbb{R}, \tilde{H}^{-1 / 2}\left(\Gamma_{i}\right)\right)}=\infty .
$$

Proof. (a) According to Lemma 4.1, the range of the operator

$$
\mathscr{N}: H_{\sigma}^{4}\left(\mathbb{R}, \tilde{H}^{-\frac{1}{2}}\left(\Gamma_{i}\right)\right) \rightarrow L_{\sigma}^{2}\left(\mathbb{R}, H^{\frac{1}{2}}\left(\Gamma_{m}\right)\right)
$$

is dense in $L_{\sigma}^{2}\left(\mathbb{R}, H^{1 / 2}\left(\Gamma_{m}\right)\right)$. For any $\varphi_{L, \mu} \in L_{\sigma}^{2}\left(\mathbb{R}, H^{1 / 2}\left(\Gamma_{m}\right)\right)$ and $\varepsilon>0$, there exists $g_{\mu, \varepsilon}^{L} \in$ $H_{\sigma}^{4}\left(\mathbb{R}, \tilde{H}^{-1 / 2}\left(\Gamma_{i}\right)\right)$ such that

$$
\left\|\mathscr{N} g_{\mu, \varepsilon}^{L}-\varphi_{L, \mu}\right\|_{L_{\sigma}^{2}\left(\mathbb{R}, H^{1 / 2}\left(\Gamma_{m}\right)\right)} \leq \varepsilon .
$$


(b) If $L \not \subset \Gamma$, we show that

$$
\lim _{\varepsilon \rightarrow 0}\left\|g_{\mu, \varepsilon}^{L}\right\|_{H_{\sigma}^{4}\left(\mathbb{R}, \tilde{H}^{-1 / 2}\left(\Gamma_{i}\right)\right)}=\infty .
$$

With the reduction to absurdity, assume that $\left\|g_{\mu, \varepsilon_{n}}^{L}\right\|_{H_{\sigma}^{4}\left(\mathbb{R}, \tilde{H}^{-1 / 2}\left(\Gamma_{i}\right)\right)}<M^{\prime}$ for an $M^{\prime}>0$ as $\varepsilon_{n} \rightarrow 0, \varepsilon_{n}>0$. Then there exists a subsequence $\varepsilon_{n}^{\prime}$ of $\varepsilon_{n}$ such that $g_{\mu, \varepsilon_{n}^{\prime}}^{L}$ converges weakly in $H_{\sigma}^{4}\left(\mathbb{R}, \widetilde{H}^{-1 / 2}\left(\Gamma_{i}\right)\right)$ to a $g \in H_{\sigma}^{4}\left(\mathbb{R}, \tilde{H}^{-1 / 2}\left(\Gamma_{i}\right)\right)$. Define

$$
\begin{aligned}
\xi & :=S_{\Gamma}^{-1} S L_{\Gamma_{i}}^{\lambda} g \in H_{\sigma}^{1}\left(\mathbb{R}, H^{\frac{1}{2}}\left(\Gamma_{i}\right)\right), \\
\varphi & :=S L_{\Gamma} \xi \in L_{\sigma}^{2}\left(\mathbb{R}, H^{\frac{1}{2}}\left(\Gamma_{m}\right)\right) .
\end{aligned}
$$

Then the continuoity of $N$ implies that $\mathcal{N} g_{\mu, \varepsilon_{n}^{\prime}}^{L} \rightarrow \varphi$ weakly in $L_{\sigma}^{2}\left(\mathbb{R}, H^{1 / 2}\left(\Gamma_{m}\right)\right)$.

Thus $\varphi_{L, \mu}=\varphi$ on $\Gamma_{m}$. This means that the Fourier-Laplace transforms $\mathscr{L}[\varphi](\cdot, \omega)$ and $\mathscr{L}\left[\varphi_{L, \mu}\right](\cdot, \omega)$ coincide on $\Gamma_{m}$ for a.e. $\omega \in \mathbb{C}_{\sigma}$. Since $\Gamma_{m}$ is a closed Lipschitz curve, the unique solvability of scattering problems at frequency $\omega \in \mathbb{C}_{\sigma}$ and the unique continuation property imply that $\mathscr{L}[\varphi](\cdot, \omega)=\mathscr{L}\left[\varphi_{L, \mu}\right](\cdot, \omega)$ in $\mathbb{R}^{2} \backslash(L \cup \Gamma)$ for a.e. $\omega \in \mathbb{C}_{\sigma}$. Hence, $\varphi_{L, \mu}=\varphi=S L_{\Gamma} \xi$ in $\left(\mathbb{R}^{2} \backslash(L \cup \Gamma)\right) \times \mathbb{R}$, i.e. $\varphi_{L, \mu} \in \operatorname{range}\left(S L_{\Gamma}\right)$ and Lemma 4.2 implies $L \subset \Gamma$. This contradicts $L \not \subset \Gamma$. The proof is complete.

In numerical experiments of this work, we employ the linear sampling method based on the near field equation (4.1) with the test function $\varphi_{z, \mu}$. However, in Theorem 4.1, the blow-up property is proved for the near field equation with the test function $\varphi_{L, \mu}$. In fact, the curve $L$ degenerates to a sampling point $z$ when the function $\zeta(x, t)$ is chosen as an appropriate delta sequence. Therefore, in computations it is reasonable to replace the test function $\varphi_{L, \mu}$ by $\varphi_{z, \mu}$.

\section{Numerical Experiments}

In this section, we demonstrate the feasibility of the RPBIE method for the forward scattering problem and the LSM for the inverse problem. We present several validating numerical examples for the cases of both single and multiple cracks with Dirichlet boundaries.

The signal function is chosen as $\zeta(t)=\lambda(t)=\sin (4 t) \mathrm{e}^{-1.6(t-3)^{2}}$. Fig. 2 shows the pulse function $\lambda(t)$ and its Fourier spectrum. The time variable should be considered and the time discretisation

$$
t_{n}=n \kappa,
$$

where $n=0,1, \ldots, N_{T}-1, \kappa=T /\left(N_{T}-1\right), N_{T}$ is the number of time steps, and $T$ the end of the recording time. The latter is chosen to make sure that the receivers obtain enough data for reconstruction. In both forward and inverse problems we choose $T=20, N_{T}=129$ and the scaling parameter $c=1$.

In the forward calculations utilising the RPBIE, we employ fully discrete Calderón calculus combined with a trapezoidal rule based convolution quadrature routine to approximate the scattered field in the time domain [13]. To illustrate the effectiveness of the RPBIE in 


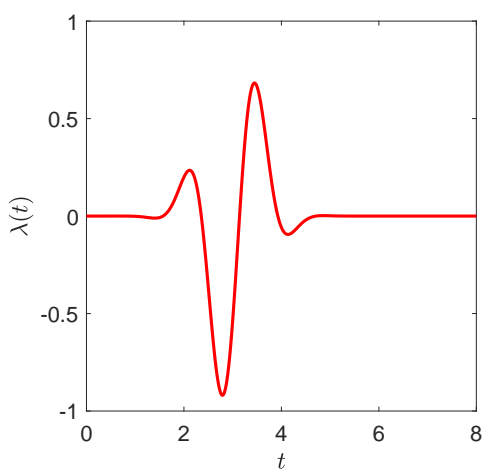

(a)

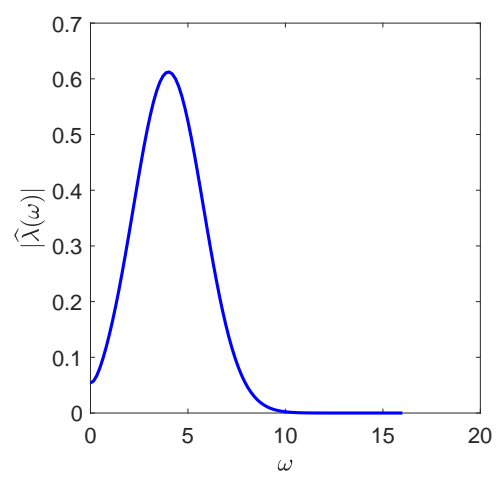

(b)

Figure 2: (a) Pulse function $t \mapsto \lambda(t)$. (b) Fourier transform of pulse function $\omega \mapsto|F(\lambda)(\omega)|$.

forward scattering problems, we take six snapshots of the scattered field. The crack is given by a half-kite-shaped open arc

$$
\Gamma_{1}:=\{\varrho(t)=(\cos t+0.65 \cos 2 t-0.65,1.5 \sin t): 0 \leq t \leq \pi\}
$$

As an incident wave, we choose the cylindrical wave with the source point located at $(-0.3920,-3.9807)$. The snapshots of the total field are shown in Fig. 3.

In order to solve the inverse problem, the scattered data are given by the forward solver

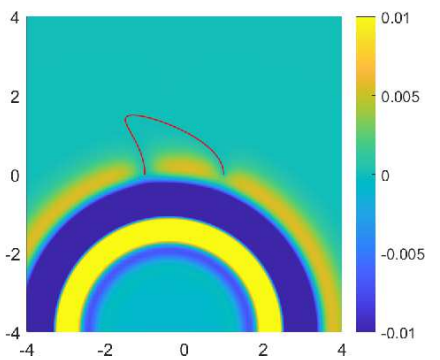

(a)

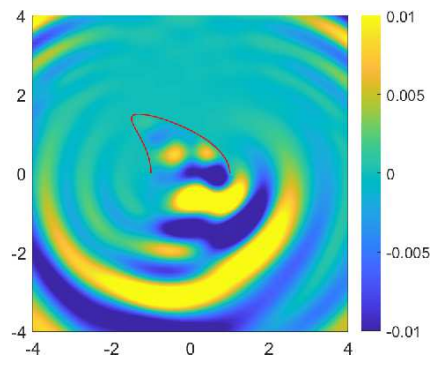

(d)

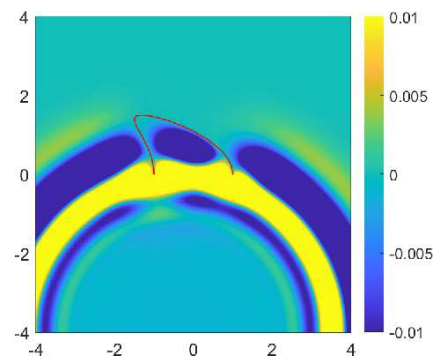

(b)

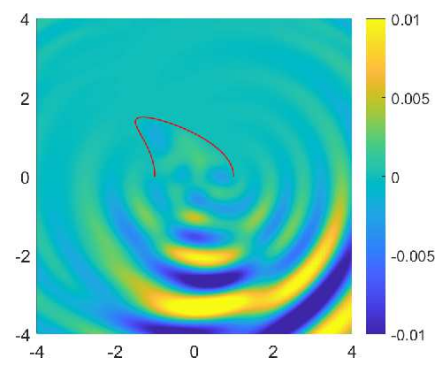

(e)

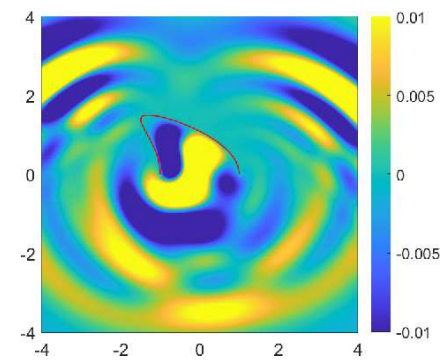

(c)

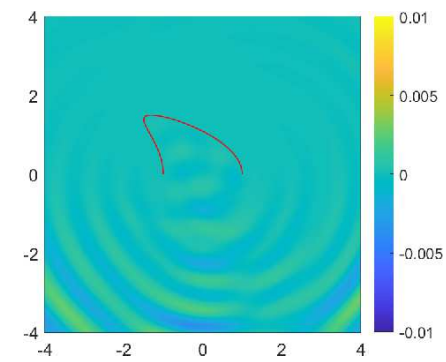

(f)

Figure 3: Snapshots of total wave field at different time steps $t_{n}$. (a) $n=42$, (b) $n=51$, (c) $n=73$, (d) $n=82$, (e) $n=90$, (f) $n=107$. Point source is located at $(-0.3920,-3.9807)$. 
and have the form

$$
u\left(x_{m}, t_{n} ; y_{i}\right), \quad m=1,2, \ldots, N_{m}, \quad n=0,1, \ldots, N_{T}-1, \quad i=1,2, \ldots, N_{i},
$$

where $\left\{y_{i}\right\} \in \Gamma_{i}$ and $\left\{x_{m}\right\} \in \Gamma_{m}$ are the positions of $N_{i}$ emitters and $N_{m}$ receivers, respectively. The near field operator $N$ and the test function $\varphi_{z, \mu}$ are discretised as follows

$$
(\mathbf{N g})(m, n)=\sum_{i=1}^{N_{i}} \sum_{j=0}^{n} u^{s}\left((n-j) \kappa, x_{m} ; y_{i}\right) \mathbf{g}(i, j)
$$

and

$$
\varphi_{z, \mu}(m, n)=\varphi_{z, \mu}\left(x_{m}, n \kappa\right)
$$

where $m=1,2, \ldots, N_{m}$ and $n=0,1, \ldots, N_{T}-1$.

The numerical inversion consists of the following steps:

1. Represent $\mathbf{N}$ in the explicit matrix form and compute the singular value decomposition of that matrix.

2. Select an appropriate time shift $\mu \in \mathbb{R}$, a sampling region $D \subset \mathbb{R}^{2} \backslash\left(\Gamma_{i} \cup \Gamma_{m}\right)$ and sampling points in the sampling region. Using the Tikhonov regularisation combined with the Morozov discrepancy, compute the regularised solution $\mathbf{g}_{z, \mu}$ of the discrete near-field equation $\mathbf{N g}_{z, \mu}=\varphi_{z, \mu}$ for each sampling point $z$.

3. Plot the contour lines of the indicator function

$$
\mathbf{G}_{\mu}(z)=\left(\sum_{j=0}^{N_{T}-1} \sum_{i=1}^{N_{i}}\left|\mathbf{g}_{z, \mu}(i, j)\right|^{2}\right)^{-\frac{1}{2}}
$$

over the sampling grid. The profile of cracks emerges on the region where the value of indicator function is relatively large.

In all numerical experiments, the emitters are distributed uniformly on the circle of radius 4 centered at the origin and receivers are located at the same points. The sampling region is the square with the side length 6 centered at the origin. The random noise

$$
u^{\epsilon}=u(1+\epsilon r)
$$

with the noise level $\epsilon$ and $r$ are random numbers in $[-1,1]$, is added to the discrete nearfield data.

In the first experiment, we compare the reconstructions by the time domain LSM developed in this work and the one given by the corresponding frequency domain counterpart. We consider the half-kite-shaped crack $\Gamma_{1}$ and the L-shaped crack with vertices $(1,0),(0,0)$ and $(0,1)$. The synthetic near-field data for the frequency domain problems are generated by the method from [12]. The results are shown in Fig. 4. 


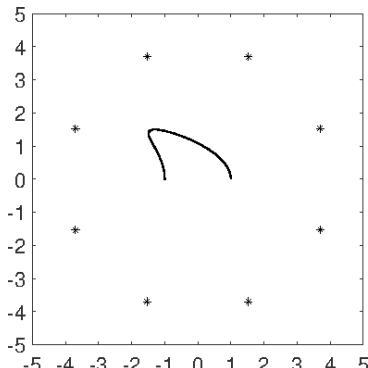

(a)

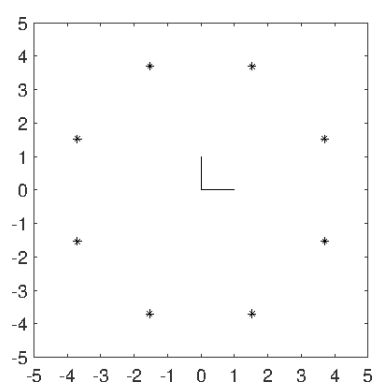

(d)

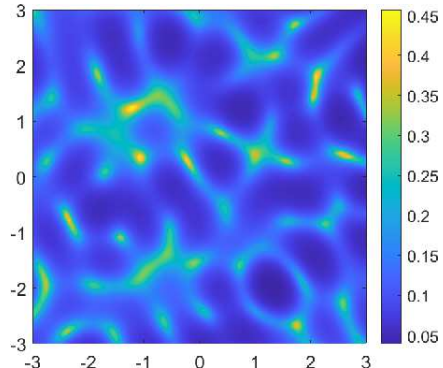

(b)

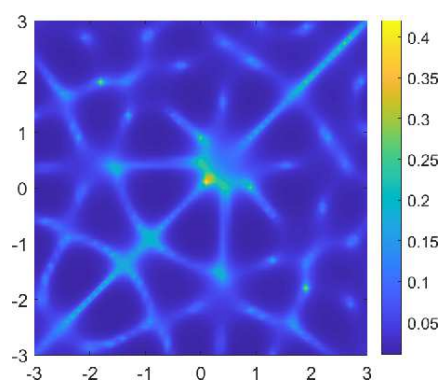

(e)

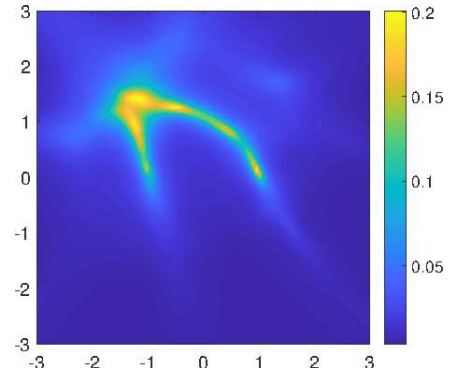

(c)

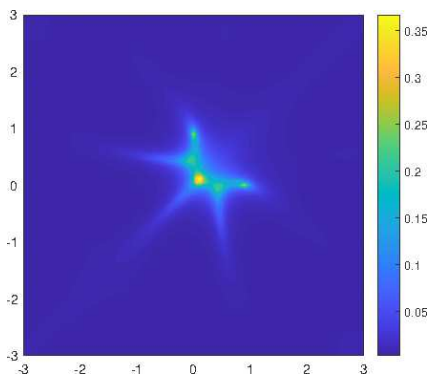

(f)

Figure 4: Comparison of time and frequency domains linear sampling methods. (a,d) True crack (solid line) and 8 emitters/receivers (small stars). (b,e) Frequency domain reconstruction with wave number $k=4$ (the central wave number for the time domain signal). (c,f) Time domain reconstruction with time shift $\mu=10$, noise level $\epsilon=1 \%$.

It is well-known that the single-frequency version of LSM provides a reasonable reconstruction only if multi-static measurements on a sufficient spatial aperture are available. However, Fig. 4 shows that the time domain LSM can still ensure acceptable reconstructions with only a few emitters and receivers used. This example indicates that the information from the temporal signal is more valuable than the frequency domain data at a single frequency.

In the second experiment, we consider the reconstruction of multiply connected cracks with different time shift parameters. As the cracks, we consider the bowl-shaped crack

$$
\Gamma_{2}:=\left\{\varrho(t)=\left(2 \sin \frac{t}{2},-\sin t\right): \frac{\pi}{4} \leq t \leq \frac{7 \pi}{4}\right\}
$$

and the segment with the vertices $(-1.369,-1.136)$ and $(-0.631,1.136)$. Fig. 5 shows that different values of $\mu$ located between 8 and 12 provide acceptable reconstructions with the best one given by $\mu=10 \mathrm{t}$.

The third experiment compares reconstructions from full aperture and limited aperture data. The scatterer profile consists of four parts - viz. the horizontal segment with vertices $(-1.9976,-2)$ and $(-1.0024,-2)$, the oblique segment with vertices $(-1.8818,1.8818)$ and $(-1.0021,1.0021)$, the parabola-shaped crack

$$
\Gamma_{3}:=\left\{\varrho(t)=0.5\left(t,\left(t^{2}-0.5\right)\right)+(1.5,1.5):-1 \leq t \leq 1\right\}
$$




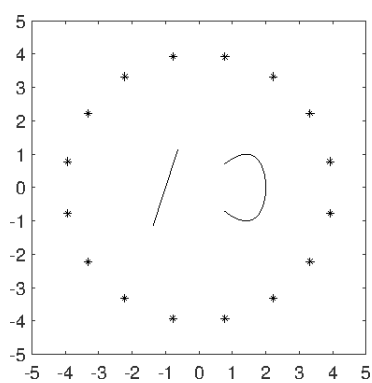

(a)

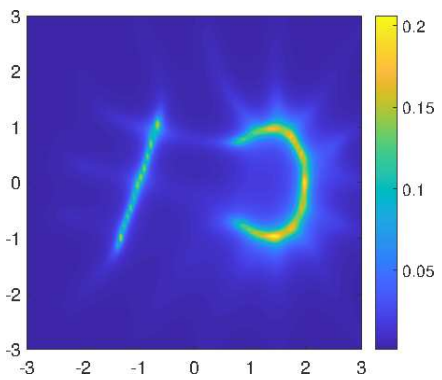

(d)

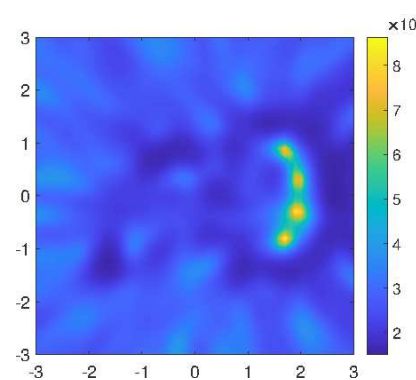

(b)

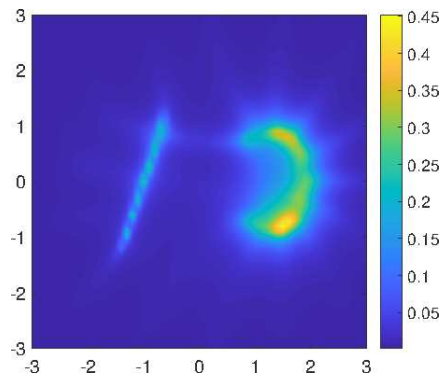

(e)

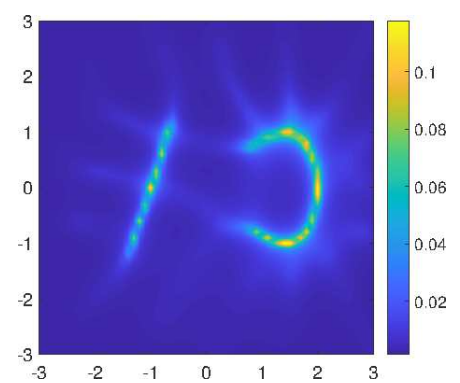

(c)

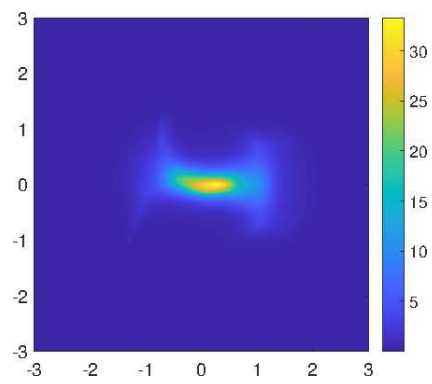

(f)

Figure 5: Influence of time shift parameter $\mu$ in time domain reconstructions, no noise added. (a) True cracks (solid line) and 16 emitters/receivers (small stars). (b) $\mu=2$, (c) $\mu=8$, (d) $\mu=10$, (e) $\mu=12$, (f) $\mu=15$.

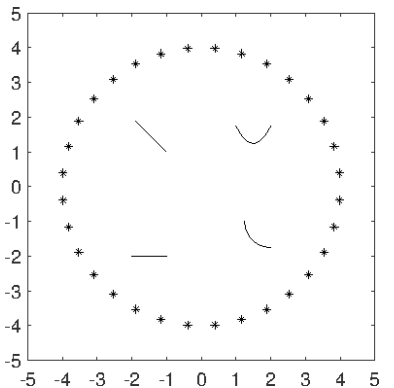

(a)

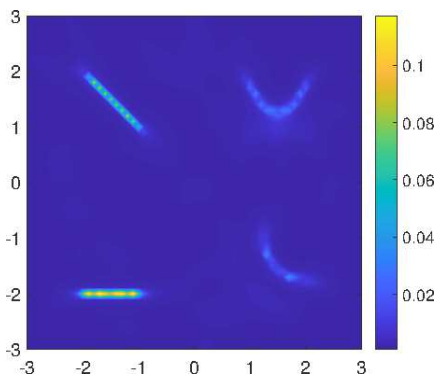

(d)

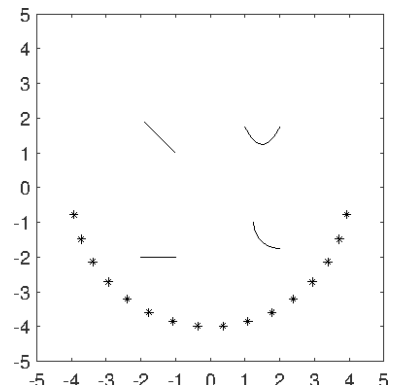

(b)

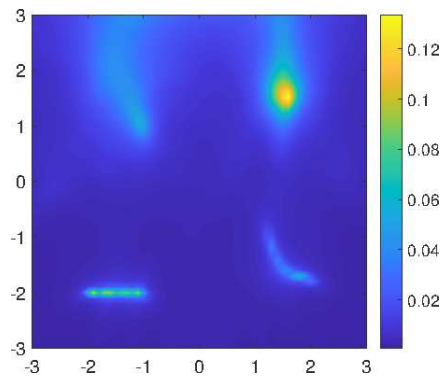

(e)

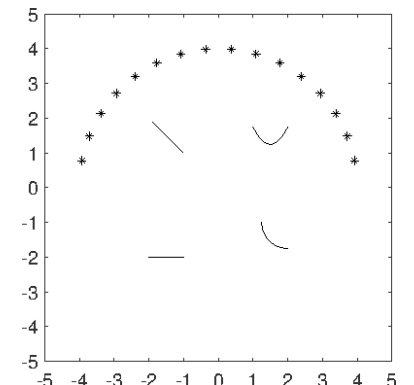

(c)

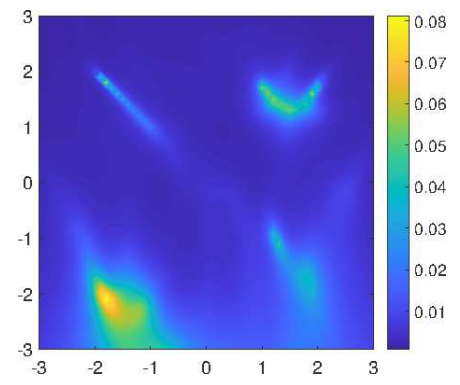

(f)

Figure 6: Reconstruction of multiple cracks from full aperture and limited aperture time domain nearfield data. (a,b,c) True cracks (solid line) and 32, 16 and 16 emitters/receivers (small stars). (d,e,f) Time domain reconstructions corresponding to (a), (b) and (c). 
and the arc

$$
\Gamma_{4}:=\left\{\varrho(t)=0.75(\cos t, \sin t)+(2,-1): \pi \leq t \leq \frac{3}{2} \pi\right\} .
$$

In order to generate the limited aperture data, emitters are first placed on the upper semicircle and then on lower one. Random noise is added with $\varepsilon=0.01$. The time shift is $\mu=10$. Fig. 6 shows that full aperture data provide better reconstruction of multiple cracks. Besides, the reconstruction of illuminated cracks is also satisfactory, even if limited aperture data are used.

\section{Acknowledgements}

The work of Fuming Ma was supported by the NSFC (No. 11771180). The work of Bo Chen was supported by the NSFC (No. 12101603) and the Scientific Research Foundation of Civil Aviation University of China (No. 2017QD04S).

\section{References}

[1] T. Arens, Why linear sampling works, Inverse Problems 1, 163-173 (2003).

[2] L. Banjai and S. Sauter, Rapid solution of the wave equation in unbounded domains, SIAM J. Numer. Anal. 1, 227-249 (2008).

[3] G. Bao, H. Liu, P. Li, and L. Zhang, Inverse obstacle scattering in an unbounded structure, Commun. Comput. Phys. 5, 1274-1306 (2019).

[4] F. Cakoni and D. Colton, The linear sampling method for cracks, Inverse Problems 2, 279-295 (2003).

[5] F. Cakoni, D. Colton, and P. Monk, The linear sampling method in inverse electromagnetic scattering, in: 80 CBMS-NSF Regional Conference Series in Applied Mathematics, SIAM (2011).

[6] B. Chen, Y. Guo, F. Ma, and Y. Sun, Numerical schemes to reconstruct three-dimensional timedependent point sources of acoustic waves, Inverse Problems 7, 075009 (2020).

[7] B. Chen, F. Ma, and Y. Guo, Time domain scattering and inverse scattering problems in a locally perturbed half-plane, Appl. Anal. 8, 1303-1325 (2017).

[8] Q. Chen, H. Haddar, A. Lechleiter, and P. Monk, A sampling method for inverse scattering in the time domain, Inverse Problems 8, 85001-85017 (2010).

[9] D. Colton and R. Kress, Integral Equation Methods in Scattering Theory, John Wiley (1983).

[10] D. Colton and R. Kress, Inverse Acoustic and Electromagnetic Scattering Theory, Springer (2013).

[11] D. Colton and P. Monk, Recent developments in inverse acoustic scattering theory, Siam Rev. 3, 369-414 (2000).

[12] V. Domínguez, S.L. Lu, and F.J. Sayas, A fully discrete Calderón calculus for two dimensional time harmonic waves, Int. J. Numer. Anal. Model. 2, 332-345 (2014).

[13] V. Domínguez, S.L. Lu, and F.J. Sayas, A Nyström flavored Calderón calculus of order three for two dimensional waves, Comput. Math. Appl. 1, 217-236 (2014).

[14] A. Geranmayeh, Time Domain Boundary Integral Equations Analysis, PhD Thesis, Technische Universität, Darmstadt (2011).

[15] Y. Guo, D. Hömberg, G. Hu, J. Li, and H. Liu, A time domain sampling method for inverse acoustic scattering problems, J. Comput. Phys. 314, 647-660 (2016). 
[16] Y. Guo, P. Monk, and D. Colton, Toward a time domain approach to the linear sampling method, Inverse Problems 9, 095016 (2013).

[17] T. Ha-Duong, On retarded potential boundary integral equations and their discretisation, in: Topics in Computational Wave Propagation, M. Ainsworth, P. Davies, D. Duncan, B. Rynne, P. Martin (Eds), pp. 301-336, Springer (2003).

[18] G.C. Hsiao and W.L. Wendland, Boundary Integral Equations, Springer (2008).

[19] A. Ishida, Propagation property and application to inverse scattering for fractional powers of negative Laplacian, East Asian J. Appl. Math. 1, 106-122 (2020).

[20] A. Kirsch and S. Ritter, A linear sampling method for inverse scattering from an open arc, Inverse Problems 1, 89-105 (2000).

[21] R. Kress, Fréchet differentiability of the far field operator for scattering from a crack, J. Inverse Ill-Posed Probl. 4, 305-313 (1995).

[22] R. Kress, Inverse scattering from an open arc, Math. Methods Appl. Sci. 4, 267-293 (1995).

[23] W. McLean, Strongly Elliptic Systems and Boundary Integral Equations, Cambridge University Press (2000).

[24] F.J. Sayas, Retarded Potentials and Time Domain Boundary Integral Equations: A Road-map, Springer Series in Computational Mathematics, Springer (2016).

[25] E.P. Stephan and W.L. Wendland, An augmented Galerkin procedure for the boundary integral method applied to two-dimensional screen and crack problems, Appl. Anal. 3, 183-219 (1984).

[26] Y. Sun, Indirect boundary integral equation method for the Cauchy problem of the Laplace equation, J. Sci. Comput. 2, 469-498 (2017).

[27] Q. Wu and G. Yan, The factorization method for an open arc, J. Comput. Math. 5, 517-532 (2015).

[28] J. Yue, M. Li, P. Li, and X. Yuan, Numerical solution of an inverse obstacle scattering problem for elastic waves via the Helmholtz decomposition, Commun. Comput. Phys. 3, 809-837 (2019). 